\title{
Applications of Surface-Enhanced Laser Desorption/Ionization Time-Of- Flight (SELDI-TOF) Mass Spectrometry in Defining Salivary Proteomic Profiles
}

\author{
Sandra K. Al-Tarawneh ${ }^{1}$ and Sompop Bencharit ${ }^{1,2, *}$
}

\author{
${ }^{I}$ Department of Prosthodontics, School of Dentistry, University of North Carolina, Chapel Hill, NC, USA and ${ }^{2}$ Depart- \\ ment of Pharmacology, School of Medicine, and the Lineberger Comprehensive Cancer Center, University of North \\ Carolina, Chapel Hill, NC, USA
}

\begin{abstract}
Recent advancement in mass spectrometry leads us to a new era of proteomic analysis. Human saliva can be easily collected; however, the complexity of the salivary proteome in the past prevented the use of saliva for proteomic analysis. Here we review the development of proteomic analyses for human saliva and focus on the use of a new mass spectrometric technology known as surface-enhanced laser desorption/ionization time-of-flight mass spectrometry (SELDI-TOF). SELDI-TOF, a modification of matrix-assisted laser desorption/ionization mass spectrometry (MALDITOF), combines the precision of mass spectrometry and the high-through-put nature of protein arrays known as Protein Chips. This technology shows a promising future for salivary proteomic analysis in monitoring treatments and diseases, as well as novel biomarker discovery.
\end{abstract}

Key Words: Proteomics, mass spectrometry, biomarker, biomarker discovery, proteomic profiling, protein expression, SELDI, SELDI-TOF, protein chips.

\section{INTRODUCTION}

Proteomic analysis involves the study of complex mixtures of proteins and their interactions. The term proteome dates back to 1994 when Marc Wilkins defined it as "the total protein content of a genome" $[1,2]$. Proteomic analysis can be viewed as an experimental approach to explain the information contained in genomic sequences in terms of the structure, function, and control of biological processes and pathways. Therefore, the proteome reflects the cellular state or the external conditions encountered by a cell. In addition, proteomic analysis can be viewed as a genome-wide assay to differentiate distinct cellular states and to determine the molecular mechanisms that control them [3]. It is now generally recognized that expression analysis directly at the protein level as well as chemical modifications of expressed proteins are necessary to unravel the critical changes that occur as part of disease pathogenesis [4].

Quantitative proteomic analyses can be used to identify the protein content in complex samples such as cell and tissue extracts or subcellular fractions, and to determine the quantitative difference in abundance for each polypeptide contained in different samples. It is expected that the proteomic profiling patterns resulting from such analyses define comprehensive molecular signatures in health and disease. Analyses of the proteomic profiles would impact a wide range of biological and clinical research questions, such as

*Address correspondence to this author at the CB \#7450, Department of Prosthodontics, School of Dentistry, University of North Carolina at Chapel Hill, Chapel Hill, NC 27599-7450, USA;

E-mail: Sompop_Bencharit@dentistry.unc.edu the systematic study of biological processes and the discovery of clinical biomarkers for detection, diagnosis, and assessment of treatment outcome. The exploitation of a proteomic approach for the study of different diseases has led to the hypothesis that multiple biomarkers or a panel of biomarkers shown by proteomic profiling may correlate more reliably with a specific disease than a single biomarker or protein [4-6].

Biomarkers can be defined as cellular, biochemical, and molecular alterations by which normal, abnormal, or simply biologic processes can be recognized or monitored. These alterations should be able to objectively measure and evaluate normal biological process, pathogenic processes, or pharmacologic responses to a therapeutic intervention. Therefore, proteomic profiling is valuable in the discovery of biomarkers as the proteome reflects both the intrinsic genetic program of the cell and the impact of its immediate environment. Protein expression and function are subject to modulation through transcription as well as through translational and posttranslational events. In addition, biomarkers can be subtle changes in molecular structures, for instance alterations of post-translation modifications, which often can only be examined at the protein level [1-6].

Currently investigators are pursuing three different approaches to develop a technology to study biomarkers with increased sensitivity and specificity. The first is to improve on currently used or known biomarkers. The second approach is to discover and validate novel biomarkers with greater sensitivity and specificity. The third approach is to use a panel of biomarkers, either by combining several indi- 
vidually identified biomarkers or by using mass spectrometry to identify a pattern of protein peaks in sera that can be used to predict the presence of cancer or other diseases. Expression pattern of a known biomarker or correlation of expression of several known biomarkers can be a valuable research and clinical tool for monitoring disease or treatment progression. High-throughput proteomic methodologies have the potential to revolutionize protein biomarker discovery and to allow for multiple markers to be assayed simultaneously. With the significant advances in mass spectrometry and proteomics technologies, protein biomarker discovery has become one of the central applications of proteomics [4$6]$.

\section{BIOMARKER DISCOVERY IN HUMAN SALIVA}

One might think tissue biopsy is the ideal specimen for disease biomarker study. However, in terms of disease diagnosis and prognosis, a human body fluid (e.g., blood, urine, or saliva) appears to be more attractive because body fluid testing provides several key advantages including low invasiveness, minimum cost, and easy sample collection and processing. Analysis of body fluid is inherently challenging because it contains a large number of proteins that could be modified in a variety of forms. Due to its complexity, a number of variables need to be considered, including sample preparation and handling, protein prefractionation, affinity depletion of highly abundant proteins, isolation of subproteomes (e.g., glycoproteome and phosphoproteome), multidimensional chromatographic separation, quantification of proteins, data analysis, database search criteria, etc. [1,3, 5-8].

Human plasma proteins originate from a variety of tissue and blood cells as a result of secretion or leakage. An issue with serum samples is sample preparation and handling. Another critical point is the complexity of the proteome $[7,8]$. Plasma/serum contains a huge number of proteins differing by the extraordinary dynamic range of at least 9-10 orders of magnitude. Many of these proteins are glycosylated or bound to other carrier proteins [7]. How to globally quantify the proteins in free, bound, or modified forms remains a critical challenge. In this regard the next logical step is to take serum screening one step further by discovering and utilizing multiple biomarkers, consisting of a pattern of upregulated and/or downregulated protein. In terms of early detection of disease progression or response to treatments, alteration of particular biomarker expression patterns may be indicated before the onset of symptoms [9].

Recently human saliva became a more attractive source for proteomic profiling. The human salivary glands produce almost $600 \mathrm{~mL} /$ day of serous and mucinous saliva containing minerals, electrolytes, buffers, enzymes and enzyme inhibitors, growth factors and cytokines, immunoglobulins (e.g., secretory immunoglobulin A [sIgA]), mucins and other glycoproteins [10-12]. Saliva plays two main roles in the biological function of the oral cavity: it is essential for the mastication, swallowing and digestion processes, and protects the teeth and the mucosal surface by means of lysozymes, cystatins, immunoglobulins and histatins which prevent the growth of microrganisms in the oral cavity [10]. In addition, the multifarious components within saliva not only protect the integrity of the oral tissues, but also provide clues to local and systemic diseases and conditions. These "salivary biomarkers" are being explored as a means of monitoring general health and in the early diagnosis of disease $[11,12]$. Indeed, human saliva has been examined in the search for biological markers of multiple systemic diseases, such as cancer, HIV, Sjögren's syndrome and cystic fibrosis [10-12].

In the past, serum has been the fluid most often used in disease diagnosis; however, saliva is a useful medium for disease diagnosis and has many advantages over both serum and urine [7,8, 10-12]. For example, salivary assays for antibodies (to viruses and bacteria), unconjugated steroid hormones (e.g., estrogen, testosterone and progesterone), environmental toxins (e.g., cadmium, lead and mercury), tobacco (nicotine) and certain drugs (e.g., ethanol, theophylline and lithium) are sufficiently sensitive to accurately reflect the blood concentrations of these substances [11,12]. In the clinic or the laboratory, saliva is relatively easy to collect in sufficient quantities for analysis, and the costs of storage and shipping tend to be lower than those for serum and urine. Noninvasiveness, and ease of sample processing are advantageous as well [7,8, 10-12]. In addition, for health care professionals and scientists, saliva tests are safer than blood tests, which are more likely to result in exposure to HIV or hepatitis [7]. On the other hand, a variety of factors may influence the rate of salivary flow and its physiologic characteristics, including circadian rhythms and activities such as exercise, and these factors should be taken into account when saliva is used as a diagnostic fluid [10-12].

Protein arrays, such as Protein Chips, are solid-phase ligand-binding assay systems using immobilized proteins on surfaces such as glass, cellulose membranes, mass spectrometer plates, microbeads, or micro/nanoparticles. The main advantages of protein arrays include high-throughput, exquisite sensitivity, and minute sample required for analysis [10]. However, the expression and purification of capture proteins, especially antibodies, is cumbersome. The design of capture arrays, particularly when screening against complex samples, also needs to take into consideration the problem of crossreactivity [7].

Considering the relatively high co-existence rate for saliva proteins and their counterpart mRNAs, the salivary transcriptome derived from DNA microarray analyses may serve as a good indicator of the diversity and range of the salivary proteome, and can be used as a reference guideline for human saliva mass spectrometry proteomic profiling [12]. For example optical fiber microarrays have been used to screen saliva from patients with end-stage renal disease (ESRD) to ascertain the efficacy of dialysis, where two salivary analytes (nitrite and uric acid) were successfully identified markers in saliva that correlate with kidney disease that were elevated in predialysis patients and were shown to be reduced following dialysis [13].

According to $\mathrm{Hu}$ et al. [12] a comparative analysis of the saliva proteome and transcriptome showed that many salivary proteins and mRNA are concordantly present in human saliva when comparison is performed within the same individual. Complementarity was obvious for common saliva proteins (such as statherin, histatins, cystatins, and prolinerich proteins), saliva enzymes (such as lysozyme, amylase, lactate dehydrogenase, and cytochrome c oxidase), and structural proteins (such as actins, tubulins, and keratins) [12]. 


\section{USE OF MASS SPECTROMETRY IN SALIVARY PROTEOMICS}

Proteomic attempts to study biological processes comprehensively by the systematic analysis of the proteins expressed in a cell or tissue. Mass spectrometry is currently proteomic's most important tool [14]. Mass spectrometry has been a powerful tool not only for protein identification in proteome analysis but also for the study of post-translational modifications of proteins, as well as chemical modification of proteins [1].

By definition, a mass spectrometer consists of an ion source, a mass analyser that measures the mass-to-charge ratio $(\mathrm{m} / \mathrm{z})$ of the ionized analytes, and a detector that registers the number of ions at each $\mathrm{m} / \mathrm{z}$ value. The mass analyser is, literally and figuratively, central to the technology [14]. There are four basic types of mass analyser currently used in proteomic research. These are the ion trap, time-of-flight (TOF), quadrupole and Fourier transform ion cyclotron analysers, and they are very different in design and performance, each with its own strength and weakness [14]. Mass spectrometric measurements are carried out in the gas phase on ionized analytes $[1,2,14]$.

During the decade of the 1990s, changes in mass spectrometric instrumentation and techniques revolutionized protein chemistry and fundamentally changed the analysis of proteins $[1-5,14]$. These changes were catalyzed by two technical breakthroughs in the late 1980s: the development of the two ionization methods electrospray ionization (ESI) and matrix-assisted laser desorption/ionization (MALDI). The ease with which proteins and peptides could be ionized by these methods rapidly made mass spectrometry a complementary technique to nuclear magnetic resonance (NMR), X-ray crystallography, circular dichroism (CD), and other classical methods of protein chemistry to study diverse aspects of protein structure and function [1,2]. For example In order to develop an ELISA respective biomarker proteins have to be purified and specific antibodies must be (commercially) available [9] and requires two antibodies for each protein that is being assayed [15] with high purity and high specificity. As a result, only a limited number of markers are currently applied for cancer detection for example. In contrast, mass spectrometry allows the multivariate analysis of complex patterns of new biomarkers without knowing their individual identities and without having specific monoclonal antibodies available. The proteins underlying individual peaks used for the differentiation do not have to be identified initially, although it may become necessary to determine the relation to the disease for a broad acceptance by the clinicians [9].

\section{MATRIX-ASSISTED LASER DESORPTION/ION- IZATION (MALDI)}

MALDI is an improvement of the laser desorption ionization (LDI) technique [1,3-6]. In LDI, a soluble analyte is air dried on a metal surface and the ionization is achieved by irradiating with an ultraviolet laser. The disadvantage of conventional LDI is that, in general, it has low sensitivity, the ionization method causes ion fragmentation, and the signal is very dependent on the ultra-violet absorbing characteristics of the analyte. This is solved with MALDI by decoup- ling the energy needed for desorption and ionization of the analyte. In MALDI, the analyte is mixed with a compound, the matrix, which absorbs the energy from the laser. The sample is co-crystallized with an excess amount of the matrix. A variety of matrices, small aromatic acids can be used. The aromatic group absorbs at the wavelength of the laser light, while the acid supports the ionization of the analyte. Irradiation with a short-pulsed laser, often a 337-nm $\mathrm{N}_{2}$ laser, causes mainly ionization of the matrix followed by energy and proton transfer to the analyte [1, 3-6].

The main advantage with MALDI ionization is high sensitivity [5-6]. Time-of-flight (TOF) mass analyzer most commonly used with MALDI is robust, simple, and sensitive and has a large mass range. MALDI-TOF mass spectra are simple to interpret due to the propensity of the method to generate predominantly singly charged ions [3-6]. However, one of the biggest problems with MALDI-TOF for identification of proteins is the difficulty in obtaining highly significant search result. This is owing to the fact that not all the expected tryptic peptides show up in the experimental MS spectrum [10]. Another problem related to the MALDI method is the heterogeneity in matrix-sample crystal formation, which makes it difficult to quantify and reproduce results [10]. Using fast evaporation methods when applying the matrix seem to give more homogenous matrix-sample crystals. However, it is still often necessary to search for a good sample spot with a target [6]. In addition, it is very time consuming, expensive, and the matrix preparation has a major effect on the quality of MALDI-TOF spectra [10]. There is no universal matrix preparation procedure that gives good results for all peptides and proteins. Only general guidelines have been found, such as the requirement of the samplematrix mixture to be adjusted to $\mathrm{pH}$ less than 2.0 for optimal signal-to-noise ratio $[5,6]$.

\section{SURFACE-ENHANCED LASER DESORPTION/ ION- IZATION (SELDI)}

The original concept of surface-enhanced laser desorption/ ionization (SELDI) was perhaps first described in 1992 by Hutchens and Yip [16-18]. Among the proteomic approaches, SELDI-TOF mass spectrometry is a highthroughput technique, particularly appropriate for the investigation of low-molecular weight proteins $(<20 \mathrm{kDa})$ with femtomole sensitivity and the ability to examine native proteins [10,16-18]. SELDI-TOF can be described as a type of MALDI-TOF mass spectrometry where the sample matrix, known as Protein Chip, has an active role in sample purification as well as the desorption/ionization step [10, 18]. This technology is based on the separation of proteins using their chemical and physical characteristics (i.e., hydrophobic, hydrophilic, acidic, basic, metal affinity) by performing a chromatographic separation of the sample to be analyzed [19]. Three major components constitute SELDI-TOF: the Protein Chip arrays, the mass analyzer, and the data analysis software. The principle of this technique is very simple. A few microliters of a sample of interest are deposited on the chromatographic surface. The Protein Chip arrays are incubated and then washed with an appropriate buffer. The proteins of interest are captured on the chromatographic surface by adsorption, partition, electrostatic interaction or affinity chromatography depending on their properties, and analyzed 
by TOF mass spectrometry. The result is a mass spectrum comprised of the mass to charge $(\mathrm{m} / \mathrm{z})$ values and intensities of the bound proteins/peptides [20]. Then, using these chromatographic surfaces, a laser desorption (LD) time-of-flight (TOF) mass spectrometer can generate an accurate protein profile of a biological sample requiring minimal amounts of sample. The protein sample may be analyzed directly or after proteolytic digestion of the adsorbed material [19]. Proteolysis of a protein after desorption can be used to identify binding sites such as antibody epitope sites [1].

While SELDI-TOF provides a unique sample preparation platform, it is similar to MALDI-TOF in that a laser is utilized for the ionization of samples that have been co-crystallized with a matrix on a target surface. Unlike MALDI-TOF target surfaces, however, the SELDI-TOF Protein Chip surfaces are uniquely designed to retain proteins from complex mixtures according to their specific properties using chromatographic-based selectivity [20].

One of the unique strengths of SELDI-TOF is its ability to analyze proteins from a variety of crude sample types, with minimal sample consumption and processing [20]. SELDI-TOF is very rapid and it can directly test native undigested biological samples. The use of different Protein Chips allows the separation/targeting of proteins in the sample, thereby reducing the complexity of the sample [19, 21]. As a result of surface affinity capture, compounds with shared physical and chemical properties are retained; thus, the various analytes are likely to have a more equal probability of becoming incorporated into the matrix crystal, which in turn reduces analyte signal suppression [18], the washing step removes most of the salts, which otherwise interfere with mass spectrometric analysis [21]. Finally, binding to the active probe surface may reveal the biomechanical property of the analyte, including chemical properties such as hydropathicity, total charge, pI, phosphorylation, glycosylation, and primary composition [17].

There are however a few drawbacks of SELDI-TOF. First, high-molecular weight salivary proteins and glycoproteins such as mucins might not be detected [10,18,21]. However, more mass peaks in the high-molecular weight range may be obtained by applying more appropriate buffers or varying the stringency of the washing steps. Second, competitive binding of high abundance non-informative proteins (like the mucins) on the chip surface may reduce the intensity of peptides/proteins of interest, particularly the low abundance ones [21]. This can be overcome by prefractionation of the saliva sample or the use of membranes with a specific cut-off. There is also concern that the protein peaks identified by SELDI-TOF and used for discriminating between cancer and control are not derived from the tumor per se but rather from the body's response to the cancer (epiphenomena) and that they may not be specific for cancer. Inflammatory conditions and benign pathologies may elicit the same bodily responses [4]. Finally, SELDI-TOF has lowmass resolution, which together with a lack of tandem mass spectrometric capabilities, limits the identification of salivary proteins [21]. In terms of reproducibility of the procedure, investigators routinely incorporate a number of approaches to ensure reproducible results. These include adding a quality control sample on each chip array, and normal- izing spectral data through commercially available or inhouse generated computer programs [10, 19-21].

\section{SELDI-TOF APPLICATIONS FOR SALIVARY PROTEOMIC STUDIES}

The application of SELDI TOF-MS technology is not limited to studies of ovarian [22], breast [23], pancreatic [24] and prostate cancers [25]. It has also been used in obtaining proteomic patterns for the diagnosis of bladder cancer from urine samples [26] and cervical cancer from laser capture microdissected captured cell lysates from tissue samples [27]. Cerebrospinal fluid (CSF) was used in finding and monitoring biomarkers for Alzheimer's disease [20, 28].

The proteins present in whole saliva are derived mainly from the salivary glands. Some proteins, however, originate from oral microorganisms, epithelial cells, crevicular fluid, leucocytes or dietary components. The relative contribution of sources other than the salivary glands to the composition of saliva will vary depending on e.g., the physiological status, method of stimulation, and the type of protein $[10,19,20]$. For decades, dental health professionals have used saliva to help assess the risk of caries by measuring its buffering capacity and bacterial content. Now, saliva is increasingly being used as an investigational aid in the diagnosis of human oral and systemic disease $[7,11,12]$. Such as Sjögren's syndrome [29], cystic fibrosis [30], sarcoidosis [31], diabetes mellitus [32] and diseases of the adrenal cortex [33]. However, unlike the SELDI-TOF analyses of plasma/serum or CSF, salivary proteomics using SELDI-TOF is still in its infantile stage. There are only handful published articles on salivary proteomic analysis using SELDI-TOF (Table 1). Most of these studies are to improve the technology and not biomarker monitoring or discovery studies.

The usefulness of saliva determinations by SELDI-TOF mass spectrometry depends on the application of preanalytical procedures adequate for the assay in saliva. Once the saliva sample is collected, it is important that the handling procedures do not affect the nature and the content of the saliva $[10,19,21]$. Most workers centrifuge the sample at $1000 \times g$, while others recommend higher centrifugation speed to remove cellular debris, bacteria and high molecular weight mucoproteins. Another point where opinions differ is the temperature to store samples if analysis is not performed immediately after sampling. In addition, slow freezing, long storage time, as well as freeze-thawing cycles may cause problems since some protein-induced precipitation may occur $[10,19,21]$.

Currently, there are four examples of human salivary proteomic studies using SELDI-TOF mass spectrometry to monitor known biomarkers or define novel biomarkers (Table 1). Two studies used SELDI-TOF in biomarker discovery. Streckfus et al. proposed the use of SELDI-TOF analysis of saliva in breast cancer biomarker discovery [35]. Ryu et al. studied the salivary protein expression profiling in Sjögren's syndrome [29]. Another two studies used SELDITOF to monitor known biomarkers [34, 36]. Imanguli et al. examined salivary proteomic profiles before and after stem cell therapy [34], and Kuwata et al. used SELDI-TOF analysis to examine the presence of bacterial lactofericin in saliva [36]. 
Table 1. Salivary Proteomics Using SELDI-TOF Mass Spectrometric Analysis

\begin{tabular}{|l|l|l|}
\hline \multicolumn{1}{|c|}{ Studies } & \multicolumn{1}{|c|}{ Type of Study } & Applications/Diseases \\
\hline \hline Papale et al. $2008[10]$ & Improvement of technology & Stem cell therapy \\
Schipper et al. $2007[19]$ & Improvement of technology & Breast cancer \\
Imanguli et al. $2007[34]$ & Monitoring proteomic profiles & Sjogren's syndrome \\
Schipper et al. $2007[21]$ & Improvement of technology & Bacterial biomarkers \\
Streckfus et al. $2006[35]$ & Biomarker discovery & Biomarker discovery \\
Ryu et al. $2006[29]$ & Monitoring lactoferricin in saliva & \\
Kuwata et al. $1998[36]$ & &
\end{tabular}

\section{CONCLUSION}

SELDI-TOF mass spectrometry combines the precision of MALDI-TOF proteomic analysis of mass spectrometry and the high-through-put nature of the protein array, Protein Chips. Despite this significant advancement and simplicity of the use of human saliva, there is only a handful clinic studies using SELDI-TOF in defining salivary proteome. This promising technology can be used to monitor known biomarkers and in novel biomarker discovery. SELDI-TOF can also be used as a tool to monitor the progress of diseases and effects of particular treatments.

\section{ACKNOWLEDGEMENT}

The University of North Carolina at Chapel Hill's Office of the Vice Chancellor for Research and Economic Development provided support for open access publication.

\section{REFERENCES}

[1] Lundblad RL. The evolution from protein chemistry to proteomics : basic science to clinical application, CRC/Taylor \& Francis Press: Boca Raton, FL 2006.

[2] Wilkins MR, Pasquali C, Appel RD, et al. From proteins to proteomes: large scale protein identification by two-dimensional electrophoresis and amino acid analysis. Biotechnology (N Y) 1996; 14: 61-5.

[3] Aebersold R, Goodlett DR. Mass Spectrometry in Proteomics. Chem Rev 2001; 101: 269-95.

[4] Srivastava S. Informatics in proteomics, CRC/Taylor \& Francis Press: Boca Raton, FL 2005.

[5] Matthiesen R. Mass spectrometry data analysis in proteomics. Humana Press: Totowa, NJ 2007.

[6] Matthiesen R, Mutenda KE. Introduction to proteomics. Methods Mol Biol 2007; 367: 1-35.

[7] Hu S, Loo JA, Wong DT. Human body fluid proteome analysis. Proteomics 2006; 6: 6326-53.

[8] Thongboonkerd V, Proteomics of human body fluids. SpringerLink, Humana Press: Totowa, NJ 2007.

[9] Pusch W, Flocco MT, Leung SM, Thiele H, Kostrzewa M. Mass spectrometry-based clinical proteomics. Pharmacogenomics 2003; 4: 463-76.

[10] Papale M, Pedicillo MC, Di Paolo S, et al. Saliva analysis by surface-enhanced laser desorption/ionization time-of-flight mass spectrometry (SELDI-TOF/MS): from sample collection to data analysis. Clin Chem Lab Med 2008; 46: 89-99.

[11] Lawrence HP. Salivary markers of systemic disease: noninvasive diagnosis of disease and monitoring of general health. J Can Dent Assoc 2002; 68: 170-4.

[12] Hu S, Li Y, Wang J, et al. Human saliva proteome and transcriptome. J Dent Res 2006; 85: 1129-33.

[13] Walt DR, Blicharz TM, Hayman RB, et al. Microsensor arrays for saliva diagnostics. Ann NY Acad Sci 2007; 1098: 389-400.
[14] Aebersold R, Mann M. Mass spectrometry-based proteomics. Nature 2003; 422: 198-207.

[15] Chen R, Pan S, Brentnall TA, Aebersold R. Proteomic profiling of pancreatic cancer for biomarker discovery. Mol Cell Proteomics 2005; 4: 523-33.

[16] Hutchens TW, Yip TT. Synthetic metal-binding protein surface domains for metal ion-dependent interaction chromatography. II. Immobilization of synthetic metal-binding peptides from metal ion transport proteins as model bioactive protein surface domains. J Chromatogr 1992; 604: 133-41.

[17] Hutchens TW, Nelson RW, Li CM, Yip TT. Synthetic metalbinding protein surface domains for metal ion-dependent interaction chromatography. I. Analysis of bound metal ions by matrixassisted UV laser desorption time-of-flight mass spectrometry. J Chromatogr 1992; 604: 125-32.

[18] Merchant M, Weinberger SR. Recent advancements in surfaceenhanced laser desorption/ionization-time of flight-mass spectrometry. Electrophoresis 2000; 21: 1164-77.

[19] Schipper R, Loof A, de Groot J, Harthoorn L, van Heerde W, Dransfield E. Salivary protein/peptide profiling with SELDI-TOFMS. Ann NY Acad Sci 2007; 1098: 498-503.

[20] Xiao Z, Prieto D, Conrads TP, Veenstra TD, Issaq HJ. Proteomic patterns: their potential for disease diagnosis. Mol Cell Endocrinol 2005; 230: 95-106.

[21] Schipper R, Loof A, de Groot J, Harthoorn L, Dransfield E, van Heerde W. SELDI-TOF-MS of saliva: methodology and pretreatment effects. J Chromatogr B Anal Technol Biomed Life Sci 2007; 847: 45-53.

[22] Jacobs IJ, Menon U. Progress and challenges in screening for early detection of ovarian cancer. Mol Cell Proteomics 2004; 3: 355-66.

[23] Bertucci F, Birnbaum D, Goncalves A. Proteomics of breast cancer: principles and potential clinical applications. Mol Cell Proteomics 2006; 5: 1772-86.

[24] Kuo SC, Gananadha S, Scarlett CJ, Gill A, Smith RC. Sporadicpancreatic polypeptide secreting tumors (PPomas) of the pancreas. World J Surg 2008; 32: 1815-22.

[25] Hellström M, Lexander H, Franzén B, Egevad L. Proteomics in prostate cancer research. Anal Quant Cytol Histol 2007; 29: 32-40.

[26] Vlahou A, Schellhammer PF, Mendrinos S, et al. Development of a novel proteomic approach for the detection of transitional cell carcinoma of the bladder in urine. Am J Pathol 2001; 158: 1491-502.

[27] Hu S, Denny P, Denny P, et al. Differentially expressed protein markers in human submandibular and sublingual secretions. Int $\mathbf{J}$ Oncol 2004; 25: 1423-30.

[28] Frankfort SV, van Campen JP, Tulner LR, Beijnen JH. Serum amyloid beta peptides in patients with dementia and age-matched non-demented controls as detected by surface-enhanced laser desorption ionisation-time of flight mass spectrometry (SELDITOF MS). Curr Clin Pharmacol 2008; 3: 144-54.

[29] Ryu OH, Atkinson JC, Hoehn GT, Illei GG, Hart TC. Identification of parotid salivary biomarkers in Sjogren's syndrome by surfaceenhanced laser desorption/ionization time-of-flight mass spectrometry and two-dimensional difference gel electrophoresis. Rheumatology 2006; 45: 1077-86.

[30] Shori DK, Asking B. Dynamics of protein and fluid secretion from the major salivary glands of rat: relevance of research findings to clinically observed defective secretion in cystic fibrosis. Pflugers Arch 2001; 443(Suppl 1): S11-6. 
[31] Beeley JA, Chisholm DM. Sarcoidosis with salivary gland involvement: biochemical studies on parotid saliva. J Lab Clin Med 1976; 88: 276-81.

[32] Rao PV, Reddy AP, Lu X, et al. Proteomic identification of salivary biomarkers of type-2 diabetes. J Proteome Res 2009; 8: 23945.

[33] Yamashita K, Takahashi M, Tsukamoto S, Numazawa M, Okuyama M, Honma S. Use of novel picolinoyl derivatization for simultaneous quantification of six corticosteroids by liquid chromatography-electrospray ionization tandem mass spectrometry. J Chromatogr A 2007; 1173: 120-8.
[34] Imanguli MM, Atkinson JC, Harvey KE, et al. Changes in salivary proteome following allogeneic hematopoietic stem cell transplantation. Exp Hematol 2007; 35: 184-92.

[35] Streckfus CF, Bigler LR, Zwick M. The use of surface-enhanced laser desorption/ionization time-of-flight mass spectrometry to detect putative breast cancer markers in saliva: a feasibility study. J Oral Pathol Med 2006; 35: 292-300.

[36] Kuwata H, Yip TT, Tomita M, Hutchens TW. Direct evidence of the generation in human stomach of an antimicrobial peptide domain (lactoferricin) from ingested lactoferrin. Biochim Biophys Acta 1998; 1429: 129-41.

(C) Al-Tarawneh and Bencharit; Licensee Bentham Open.

This is an open access article licensed under the terms of the Creative Commons Attribution Non-Commercial License (http: //creativecommons.org/licenses/ by-nc/3.0/) which permits unrestricted, non-commercial use, distribution and reproduction in any medium, provided the work is properly cited. 